\title{
Predicting invasive plants in California
}

by Elizabeth D. Brusati, Douglas W. Johnson and
Joseph M. DiTomaso

Preventing plant invasions or eradicating incipient populations is much less costly than confronting large well-established populations of invasive plants. We developed a preliminary determination of plants that pose the greatest risk of becoming invasive in California, primarily through the horticultural industry. We identified 774 species that are invasive elsewhere in Mediterranean climates but not yet invasive in California. From this list, we determined which species are sold through the horticulture industry, whether they are sold in California and whether they have been reported as naturalized in California. We narrowed the list to 186 species with the greatest potential for introduction and/or invasiveness to California through the horticultural trade. This study provides a basis for determining species to evaluate further through a more detailed risk assessment that may subsequently prevent importation via the horticultural pathway. Our results can also help land managers know which species to watch for in wildlands.

P lants have been transported around the world for centuries, as agricultural commodities, ornamental species or inadvertent contaminants of imported materials. Naturalized plants are those that have spread out of cultivated areas, including gardens, into more wild areas, and invasive plants are the subset of naturalized species that cause ecological or economic harm. In general, only a small proportion of plants introduced into a new region have been invasive plants. However, the number of invasive plants with horticultural origin is high, making it critically important to natural resource managers, ecologists and policymakers to predict which newly introduced species pose the greatest risk of escape and invasion.

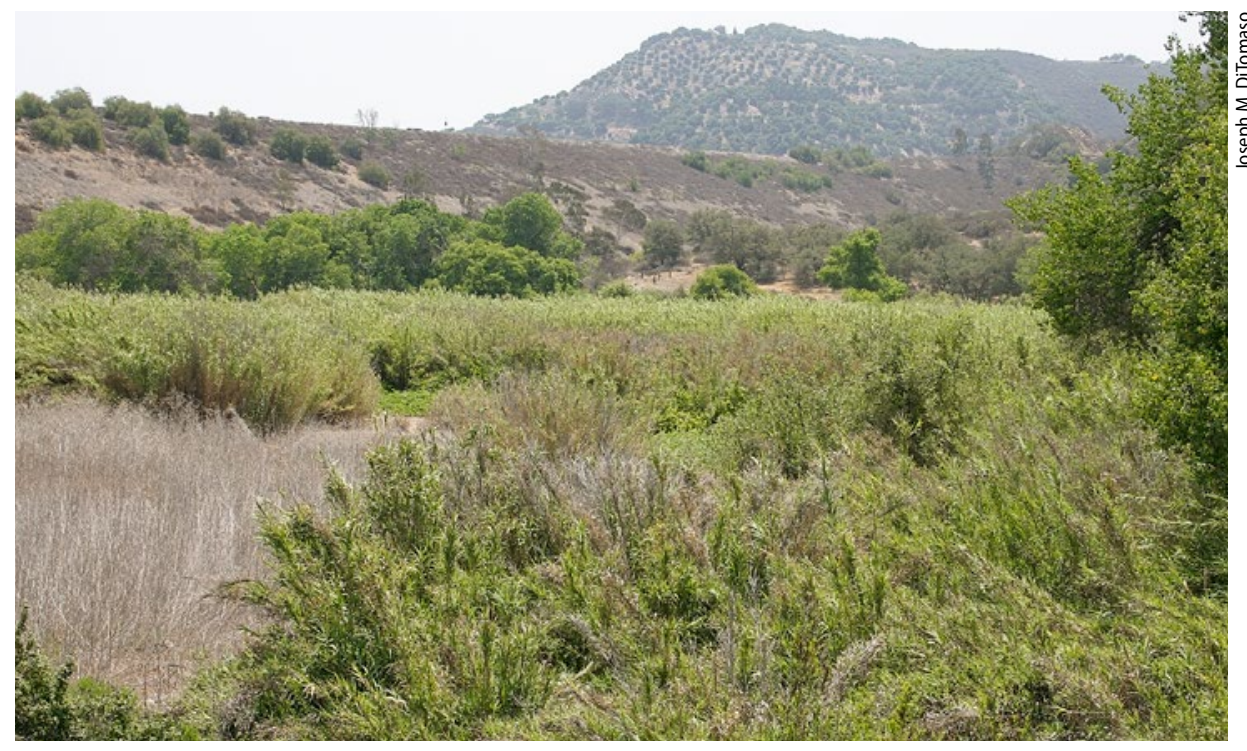

Giant reed (Arundo donax) infesting a wetland area in Southern California. Giant reed was introduced as both an ornamental and erosion control species and is now one of the most invasive species in the state.

The geographic diversity of California has led to broad evolution in native plants. California has approximately 3,400 species of native plants, of which $24 \%$ are found only in the state (Baldwin et al. 2012). However, California is also something of a hotspot for nonnative plants, with over 1,500 nonnative species naturalized, weedy in agricultural systems or invasive in natural areas (DiTomaso and Healy 2007). As a result, California not only faces a high risk of escape, establishment and invasion of introduced ornamental plants, but also has a high proportion of native species threatened by invasive plants.

Within California, there are two lists that identify invasive plants. First, based on 13 questions that assess impacts, invasiveness and distribution, the California Invasive Plant Council's list includes 214 species that cause ecological harm in the state's wildlands (Cal-IPC 2013). Approximately $63 \%$ of these species were deliberately introduced to California, mostly as ornamental plants (Bell et al. 2007). Second, the California Department of Food and Agriculture (CDFA) Noxious Weed List primarily lists plants that cause, or have the potential to cause, economic damage to the state's agricultural industry; CDFA has legal authority to regulate plants on this list through Section 4500 of the California Code of Regulations (CDFA 2013). Because the criteria for these lists have a different focus, the listed species overlap but are not the same. Few species derived from the horticultural trade are included on the state Noxious Weed List.

The high number of invasive plants with horticultural origin makes it critically important to natural resource managers, ecologists and policymakers to predict which newly introduced species pose the greatest risk of escape and invasion.

The horticultural trade is one of the major pathways for invasive plants in California and elsewhere (Drew et al. 2010; Okada et al. 2007; Reichard and White 2001). For example, higher market frequency (as measured by availability in seed catalogs) and lower prices were shown to be good predictors of a plant's probability of invasion in Britain (DehrenSchmutz et al. 2007). Horticulture is also

Online: http://californiaagriculture.ucanr.edu/ landingpage.cfm?article=ca.v068n03p89\&fulltext=yes doi: 10.3733/ca.v068n03p89 


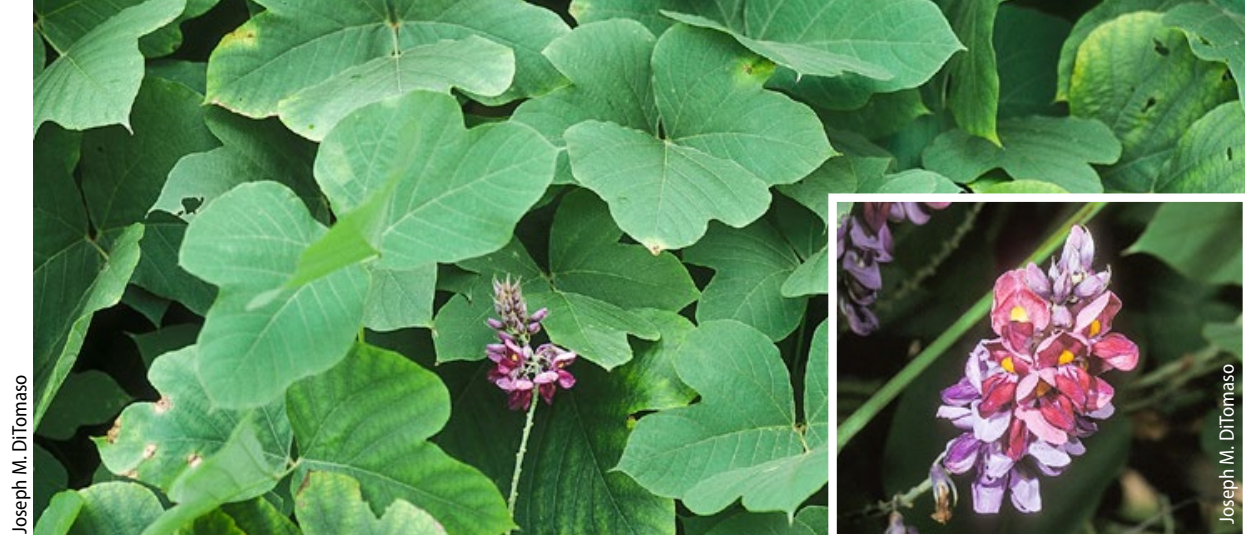

After being introduced as an animal forage species, kudzu (Pueraria montana) escaped to invade forested areas in the southern United States. Kudzu is neither naturalized nor sold in California.

a major agricultural sector in California, accounting for $\$ 2.5$ billion in sales in 2011 (CDFA 2012).

The ability to predict potential invasiveness is important both for species that have already been introduced to a region but are not yet invasive and for species that may be introduced through the horticultural industry in the future. In both cases, prediction of invasiveness before it occurs can, through collaborative efforts with the nursery industry, lead to voluntary restrictions in sales, preventing the potential for damage should the species escape cultivation.

Knowing that a plant is invasive in one region can give insight into whether it might be problematic in another region, particularly if the two regions have similar climates. For woody ornamental species, for example, being invasive elsewhere was the single best predictor of potential invasiveness in a new region of introduction (Reichard and Hamilton 1997). In addition, Caley and Kuhnert (2006) showed that four variables were most important for screening potential invasive plants: human dispersal, naturalized elsewhere, invasiveness elsewhere and a high degree of domestication. Two of these variables, human dispersal and high degree of domestication, are characteristics of horticultural species.

California is one of five Mediterranean climate regions in the world, along with the Mediterranean Basin of Europe and northern Africa, central Chile, the Cape Region of South Africa and western Australia. All these regions are characterized by a winter rainy season and a summer dry season and are likely to share invasive species due to their similar climates.

The primary objective of this study was to identify ornamental species at high risk of becoming newly invasive in California. To develop this list, we considered the single most important factor to be a species' invasiveness in other areas of the world with a similar Mediterranean climate or in a state neighboring California. While we recognize that this list is not comprehensive, we believe that it provides a good starting point for subsequently conducting risk assessments that could reduce the threat of introducing new invasive ornamentals to the state. This approach might also help determine which naturalized species should be monitored to see if they will become truly invasive.

\section{Identifying potential invaders}

Invasive plant data were collected through online databases and published lists from other regions with Mediterranean climates. We also used in California since before 1940 without becoming invasive. established invasive plants reported from states neighboring California, including Arizona (Northam et al. 2005), Nevada (Nevada Department of Agriculture 2005) and Oregon (Oregon Department of Agriculture 2006). We included species on the California Noxious Weed List (CDFA 2007) as well as those that have been shown to invade wildlands (Cal-IPC 2013; personal communications with land managers in California).

Of the plants that have invaded other Mediterranean regions, we first removed species native to California and those already known to be invasive in wildland areas within the state. Then for each of the remaining plant species, we evaluated the Mediterranean-type region(s) invaded, location of origin, human uses (especially in horticulture) and whether the species was native, cultivated, naturalized or invasive in California (Baldwin et al. 2012; Cal-IPC 2013). For species already naturalized but not yet invasive in California, we determined the year they were first reported as naturalized based on the online Consortium of California Herbaria database (ucjeps.berkeley.edu/consortium/). In addition, we determined if plants are currently sold in the horticultural and ornamental trade in California using the

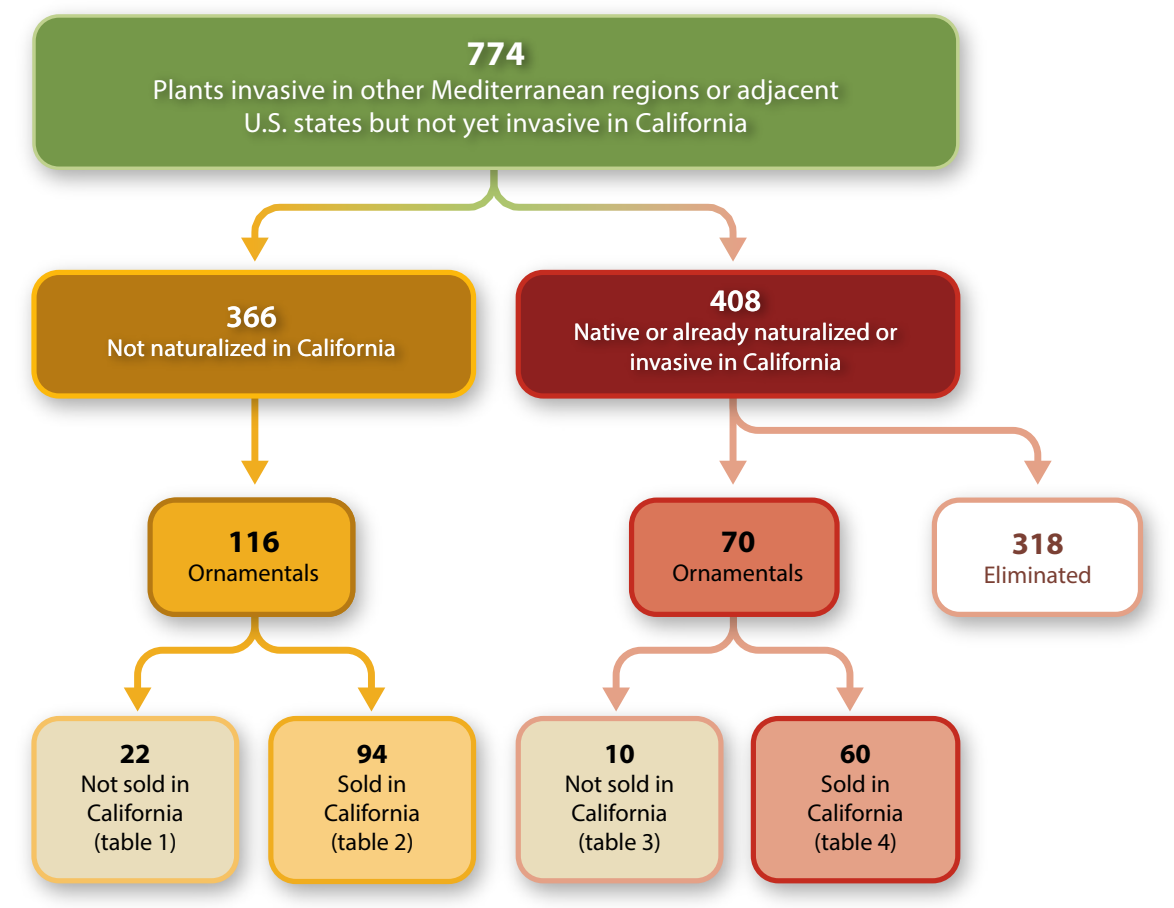

Fig. 1. Process used to determine the species with potential to become invasive in California through surveys of other Mediterranean climatic regions and adjacent U.S. states, with a focus on species sold as ornamentals. Tables 1 to 4 list these 186 species $(22+94+10+60)$. The 318 eliminated species were eliminated because they are native to California, already invasive in California or have been naturalized 
Sunset Western Garden Book (Brenzel 2007) and the Plant Locator (Hill and Narizny 2004), a directory of nurseries stocking particular species. While these references do not include all of the species available by mail order or via the Internet, they represent plants most commonly available in nurseries.

\section{Which plants are likely threats?}

Based on our criteria, we found 774 plants listed as invasive in other Mediterranean regions or adjacent states (fig. 1). Of these, 366 (47\%) are not naturalized in California and therefore fit our focus on potential new invaders. Of the remaining 408 species (53\%), we eliminated 318 species that did not fit our focus on new invaders: they were either native to California (Baldwin et al. 2012) or already invasive in California (DiTomaso and Healy 2007), or had naturalized in the state before 1940 without becoming invasive (Consortium of California Herbaria 2008). This left us with 90 species that naturalized after 1940.

We assumed that species that naturalized before 1940 and that have not yet become invasive in California are unlikely to become invasive in the future. Many of the naturalized species have been present in the state for over a century, with 20 recorded in the 1860s and 144 recorded before 1900 . While we believe that 70 years of naturalization without significant spread and harm is sufficient to consider a species as having low potential for invasion, this may not be true for all species. There may be some instances where longer lag periods - a length of time when a species is present in natural areas before beginning to spread and cause ecological harm - could occur prior to rapid expansion of a species. Furthermore, the movement of ornamental plants is facilitated by humans, thus increasing the opportunity for introduction to suitable habitats. In addition to possibly increasing the potential for invasion by introduced plants, this facilitation could also reduce the time between introduction and invasion.

Next, we subdivided the 90 species that became naturalized after 1940 and the 366 species that are not naturalized in California based on whether they are sold as ornamentals. We also noted whether they are sold in California (fig. 1). Of the 90 naturalized species, $70(78 \%)$ are currently sold as ornamentals somewhere in the world, with $60(67 \%)$ sold in California. Of the 366 nonnaturalized species in California, only 32\% (116 species) were ornamentals. The majority of these species $(94$, or $81 \%$ ) are currently sold in California, while the other 22 are ornamentals not sold in the state. Thus, in total, we listed 186 species of ornamentals as the greatest concern for introduction and/or invasiveness to California through the horticultural pathway. This total

\section{EUCALYPTUS Fuel Dynamics, and Fire Hazard in the Oakland Hills}

\begin{abstract}
Eucalyptus trees were introduced to California from Australia in the 1850 s and have become invasive in some coastal areas since then. In 1973, following a two-year study of eucalyptus stand densities, caloric content of fuel and dynamics of fuel accumulation in the Oakland Hills, researchers recommended a fuel reduction program. Eighteen years later, a firestorm in the Oakland Hills fueled by high winds and dense groves of freeze-damaged eucalyptus and pine trees killed 25 people and destroyed nearly 4,000 dwellings.
\end{abstract}

1973 "Eucalyptus has been a scenic and aromatic addition to the California landscape for over a century. The rapid growth of early plantations caught the eye of timber speculators around 1900 and millions of eucalyptus seedlings, predominately blue gum (Eucalyptus globulus) were planted. They soon covered the crest of the Berkeley-Oakland Hills, and have created a serious fire hazard since that time at the urban-wildland interface.

“... The late 1972 freeze has resulted in a proposed fuel management program for the Berkeley-Oakland Hills. Management of eucalyptus groves is an integral part of such a program. The results of this study indicate that fuel buildup occurs very rapidly in unmanaged eucalyptus stands, and to maintain low fuel levels a fuel reduction program should be implemented."

Agee JK, et al. 1973. Eucalyptus fuel dynamics, and fire hazard in the Oakland hills. Calif Agr 27(9):13-5.

Of the article's four co-authors, the two research assistants went on to distinguished professorial careers in forestry and ecological sciences, James K. Agee at the University of Washington College of Forest Resources and Ronald H. Wakimoto at the University of Montana, Missoula.

Ellis F. Darley was a plant pathologist at UC Riverside and did pioneering work on the effects of air pollution on plants and on the overall environment. At UC Berkeley, Harold H. Biswell was professor of forestry and an early proponent of controlled burning for wildland fuel management. When he retired in 1973, UC awarded him the Berkeley Citation, its highest honor for distinguished achievement. In 1994, a symposium on "Fire Issues and Solutions in Urban Interface and Wildland Ecosystems" was held in his honor.

$$
\text { -W. J. Coats }
$$

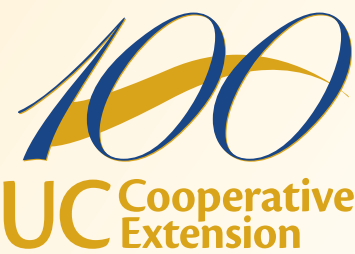

A Celebration of Science and Service 


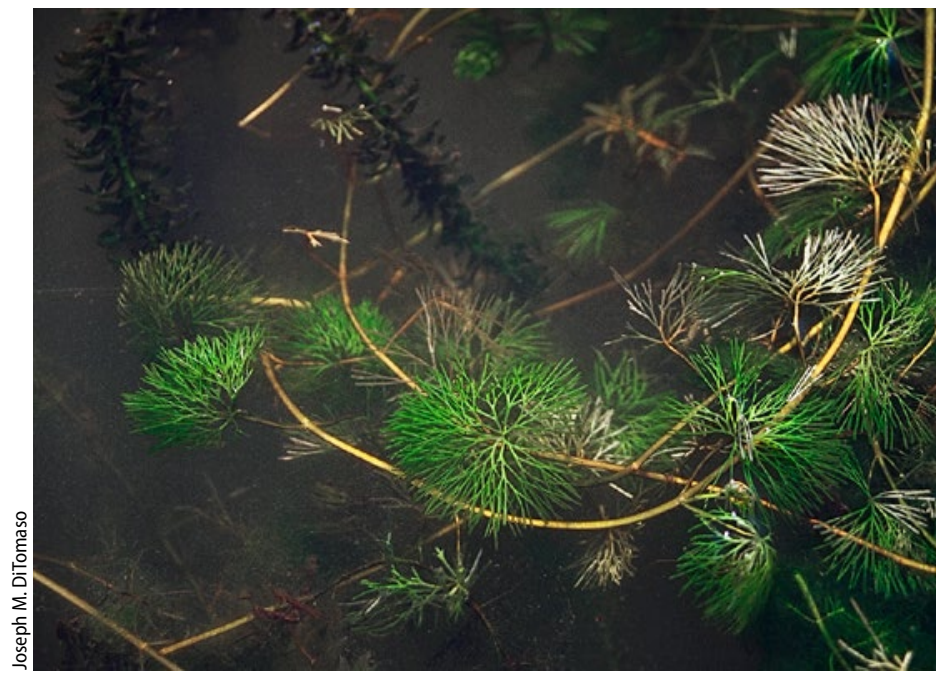

Fanwort (Cabomba caroliniana) is an invasive aquatic weed in California that was introduced through the aquarium industry.

TABLE 1. Species neither naturalized nor sold in California, but sold as ornamental plants elsewhere*

\begin{tabular}{|c|c|c|}
\hline Family & Species & Common name \\
\hline \multirow[t]{4}{*}{ Asteraceae } & $\begin{array}{l}\text { Ageratina riparia (Regel) King \& } \mathrm{H} \text {. } \\
\text { Rob. }\end{array}$ & Creeping croftonweed \\
\hline & $\begin{array}{l}\text { Gymnocoronis spilanthoides (D.Don) } \\
\text { DC. }\end{array}$ & Senegal tea \\
\hline & Senecio angulatus L.f. & Creeping groundsel \\
\hline & $\begin{array}{l}\text { Sphagneticola trilobata (L.C. Rich.) } \\
\text { Pruski }\end{array}$ & Wedelia \\
\hline Crassulaceae & Kalanchoe pinnata (Lam.) Pers. & Cathedral bells \\
\hline \multirow[t]{5}{*}{ Fabaceae } & Acacia nilotica (L.) Willd. ex Delile & Gum arabic tree \\
\hline & Albizia lebbeck (L.) Benth. & Woman's tongue \\
\hline & Caesalpinia decapetala (Roth) Alston & Shoofly \\
\hline & Pueraria montana (Lour.) Merr. & Kudzu \\
\hline & $\begin{array}{l}\text { Senna pendula (Humb. \& Bonpl. ex } \\
\text { Willd.) Irwin \& Barneby }\end{array}$ & Valamuerto \\
\hline \multirow[t]{5}{*}{ Iridaceae } & Gladiolus undulatus L. & Wild gladiolus \\
\hline & Moraea flaccida (Sweet) Steud. & One-leaf Cape tulip \\
\hline & $\begin{array}{l}\text { Moraea lewisiae (Goldblatt) } \\
\text { Goldblatt (= Hexaglottis lewisiae } \\
\text { Goldblatt) }\end{array}$ & Cape tulip \\
\hline & Sparaxis bulbifera (L.) Ker Gawl. & Wandflower \\
\hline & $\begin{array}{l}\text { Watsonia versfeldii J.W. Mathews \&. } \\
\text { L. Bolus }\end{array}$ & Bugle-lily \\
\hline Meliaceae & Toona ciliata Roem. & Australian redcedar \\
\hline Polygalaceae & Polygala virgata Thunb. & Purple broom \\
\hline Polygonaceae & $\begin{array}{l}\text { Rumex sagittatus Thunb. [= Acetosa } \\
\text { sagittata (Thunb.) L.A.S. Johnson \& } \\
\text { B.G. Briggs] }\end{array}$ & $\begin{array}{l}\text { Rambling dock, garden } \\
\text { sorrel }\end{array}$ \\
\hline Proteaceae & Hakea gibbosa (Sm.) Cav. & Hairy or rock hakea \\
\hline Salicaceae & Salix fragilis $\mathrm{L}$. & Crack willow \\
\hline Sapindaceae & Cardiospermum grandiflorum Sweet & Showy balloonvine \\
\hline Solanaceae & Cestrum laevigatum Schltdl. & Inkberry \\
\hline
\end{tabular}

* Scientific and common names of nonweedy species in all tables are from the United States Department of Agriculture Plant Database (http://plants.usda.gov/) or Germplasm Resources Information Network (www.ars-grin.gov/npgs/aboutgrin.html). Plants considered naturalized in California wildlands are based on Baldwin et al. (2012). These tables do not include species that have been present in California since before 1940 without becoming invasive. includes both those species currently sold and those that could be sold in the future (tables 1 to 4 ).

This study, however, did not take into consideration the potential effects of climate change on habitat suitability and plant invasions within California. It is possible that warmer temperatures or modified precipitation patterns due to climate change will allow some currently noninvasive ornamentals to spread and become invasive. However, predictions of the spread of invasive plants in the western United States indicate that

TABLE 2. Species sold as ornamentals in California but not yet naturalized in the state*

\begin{tabular}{|c|c|c|}
\hline Family & Species & Common name \\
\hline Acanthaceae & Thunbergia grandiflora Roxb. & $\begin{array}{l}\text { Thunbergia, Bengal } \\
\text { trumpet }\end{array}$ \\
\hline Aceraceae & Acer pseudoplatanus L. & Sycamore maple \\
\hline \multirow[t]{2}{*}{ Agavaceae } & Agave sisalana Perrine & Sisal hemp \\
\hline & Yucca gloriosa L. & Moundlily yucca \\
\hline Aloaceae & Aloe vera (L.) Burm. f. & Barbados aloe \\
\hline \multirow[t]{2}{*}{ Asclepiadaceae } & $\begin{array}{l}\text { Cryptostegia grandiflora (Roxb. ex } \\
\text { R. Br.) R. Br. }\end{array}$ & Palay rubbervine \\
\hline & Periploca graeca $\mathrm{L}$. & Silkvine \\
\hline Asparagaceae & Asparagus africanus Lam. & African asparagus \\
\hline
\end{tabular}

(formerly

Liliaceae)

\begin{tabular}{|c|c|c|}
\hline \multirow[t]{4}{*}{ Asteraceae } & Baccharis halimifolia $\mathrm{L}$. & Eastern baccharis \\
\hline & Coleostephus myconis (L.) Reichenb. & Corn marigold \\
\hline & $\begin{array}{l}\text { Schkuhria pinnata (Lam.) Kuntze } \\
\text { ex Thell. }\end{array}$ & Pinnate false-threadleaf \\
\hline & Solidago chilensis Meyen & Brazilian arnica \\
\hline Balsaminaceae & Impatiens glandulifera Royle & $\begin{array}{l}\text { Balsam, policeman's } \\
\text { helmet }\end{array}$ \\
\hline Bignoniaceae & Spathodea campanulata P. Beauv. & African tuliptree \\
\hline Boraginaceae & Echium vulgare $\mathrm{L}$. & Blueweed \\
\hline \multirow[t]{8}{*}{ Cactaceae } & $\begin{array}{l}\text { Echinopsis spachiana (Lem.) } \\
\text { Friedrich \& G.D. Rowley }\end{array}$ & Echinopsis, golden torch \\
\hline & Harrisia martini (Labouret) Britt. & Mooncactus \\
\hline & $\begin{array}{l}\text { Opuntia fulgida Engelm. } \\
\text { [=Cylindropuntia fulgida (Engelm.) } \\
\text { F.M. Knuth] }\end{array}$ & Jumping cholla \\
\hline & Opuntia humifusa Raf. & Spreading pricklypear \\
\hline & $\begin{array}{l}\text { Opuntia imbricata (Haw.) DC. [= } \\
\text { Cylindropuntia imbricata (Haw.) } \\
\text { F.M. Knuth] }\end{array}$ & $\begin{array}{l}\text { Walkingstick cholla, tree } \\
\text { cholla }\end{array}$ \\
\hline & $\begin{array}{l}\text { Opuntia microdasys (Lehm.) N.E. } \\
\text { Pfeiffer }\end{array}$ & $\begin{array}{l}\text { Angel's-wings, bunny } \\
\text { ears }\end{array}$ \\
\hline & $\begin{array}{l}\text { Opuntia robusta J.C. Wendl. ex } \\
\text { Pfeiff. }\end{array}$ & Wheel cactus, silver dollar \\
\hline & Opuntia stricta (Haw.) Haw. & Erect pricklypear \\
\hline Cannabaceae & Humulus japonicus Sieb. \& Zucc. & Japanese hops \\
\hline Cannaceae & Canna indica L. & Indian shot \\
\hline Caprifoliaceae & Leycesteria formosa Wall. & Himalayan honeysuckle \\
\hline Casuarinaceae & $\begin{array}{l}\text { Casuarina equisetifolia L. ex J.R. \& } \\
\text { G. Forst. }\end{array}$ & Australian-pine \\
\hline Convolvulaceae & Turbina corymbosa (L.) Raf. & Christmasvine \\
\hline
\end{tabular}

Asparagus plumosus (Kunth) Jessop Common asparagus fern Asparagus scandens Thunb. Climbing asparagus fern Baccharis halimifolia L. Eastern baccharis Coleostephus myconis (L.) Reichenb. Corn marigold Schkuhria pinnata (Lam.) Kuntze

Solidago chilensis Meyen

Balsam, policeman's helmet

African tuliptree

Blueweed

Boraginaceae

Echinopsis spachiana (Lem.)

Friedrich \& G.D. Rowley

Opuntia fulgida Engelm.

$=$ Cylindropuntia fulgida (Engelm.)

Opuntia imbricata (Haw.) DC. [= ylindropuntia imbricata (Haw.)

Pfeiffer

Opuntia robusta J.C. Wendl. ex Opuntia stricta (Haw.) Haw.

Erect pricklypea

Japanese hops

Caprifoliaceae

. 
while some will likely spread, others may contract their ranges (Bradley et al. 2009). Thus, it was not possible to determine the impact of climate change on all the species evaluated in this study.

\section{Management implications}

To reduce the sale of invasive plants in California, environmental groups, scientists, government agencies and the horticulture industry are participating in the PlantRight partnership, a coalition that works with retail nurseries and growers on voluntary measures to reduce the sale of invasive plants and promote noninvasive alternatives (plantright.org); the authors serve on its steering committee. Specific guidelines or recommendations could be established for the high-risk species we identified in tables 1 to 4 to minimize future introduction, establishment and invasion. Cooperative efforts can discourage the introduction of ornamental plants in other regions that are neither naturalized nor sold in California (table 1), and these plants also could be included in a cautionary list that would require

TABLE 2. Continued from previous page

\begin{tabular}{|c|c|c|c|c|c|}
\hline Family & Species & Common name & Family & Species & Common name \\
\hline \multirow[t]{2}{*}{ Dryopteridaceae } & Nephrolepis cordifolia (L.) C. Presl & Narrow swordfern & \multirow[t]{2}{*}{ Pinaceae } & Pinus canariensis C. Sm. & Canary Island pine \\
\hline & Nephrolepsis exaltata (L.) Schott & Swordfern, Boston fern & & Pinus elliottii Engelm. & Slash pine \\
\hline Ericaceae & Erica arborea L. & Briar root, tree heath & & Pinus nigra Arnold & Austrian pine \\
\hline Euphorbiaceae & Euphorbia polygonifolia (L.) Small & Seaside sandmat & & \multirow{2}{*}{$\begin{array}{l}\text { Pinus patula Schiede ex Schltdl. \& } \\
\text { Cham. }\end{array}$} & \multirow{2}{*}{$\begin{array}{l}\text { Jelecote pine, Mexican } \\
\text { weeping pine }\end{array}$} \\
\hline \multirow[t]{11}{*}{ Fabaceae } & Acacia karroo Hayne & Karroothorn & & & \\
\hline & \multirow{2}{*}{$\begin{array}{l}\text { Acacia podalyriifolia A. Cunn. ex } \\
\text { G. Don }\end{array}$} & \multirow[t]{2}{*}{ Pearl wattle } & & Pinus pinaster Aiton & Maritime pine \\
\hline & & & \multirow[t]{2}{*}{ Poaceae } & Glyceria maxima (Hartm.) Holmb. & Reed mannagrass \\
\hline & Acacia stricta (Andrews) Willd. & Hop wattle & & Paspalum vaginatum Sw. & Seashore paspalum \\
\hline & Cassia fistula $\mathrm{L}$. & Golden shower, senna & Polygonaceae & Polygonum campanulatum Hook. f. & Bellflower smartweed \\
\hline & Dalbergia sissoo Roxb. ex DC. & $\begin{array}{l}\text { Indian rosewood, } \\
\text { Himalayan raintree }\end{array}$ & Proteaceae & $\begin{array}{l}\text { Hakea drupacea (C.F. Gaertn.) } \\
\text { Roem. \& Schult. }\end{array}$ & Sweet hakea \\
\hline & \multirow[t]{2}{*}{ Psoralea pinnata $\mathrm{L}$. } & \multirow{2}{*}{$\begin{array}{l}\text { Blue psoralea, } \\
\text { fountainbush }\end{array}$} & & Hakea salicifolia (Vent.) B.L. Burtt & Willow-leaved hakea \\
\hline & & & & Hakea sericea Schrad. \& J.C. Wendl & Needlebush, silky hakea \\
\hline & $\begin{array}{l}\text { Retama raetum (Forssk.) Webb \& } \\
\text { Berthel. }\end{array}$ & Weeping white broom & \multirow[t]{2}{*}{ Rhamnaceae } & Rhamnus alaternus $\mathrm{L}$. & Italian buckthorn \\
\hline & Senna alata (L.) Roxb. & $\begin{array}{l}\text { Emperor's candlesticks, } \\
\text { candlebush }\end{array}$ & & Ziziphus mauritiana Lam. & $\begin{array}{l}\text { Indian jujube, Chinese } \\
\text { apple }\end{array}$ \\
\hline & Senna bicapsularis (L.) Roxb. & Christmasbush & \multirow[t]{2}{*}{ Rosaceae } & $\begin{array}{l}\text { Cotoneaster divaricatus Rehder \& } \\
\text { E.H. Wilson }\end{array}$ & Spreading cotoneaster \\
\hline Fagaceae & Quercus robur L. & English oak & & Cotoneaster glaucophyllus Franch. & Cotoneaster \\
\hline \multirow[t]{4}{*}{ Iridaceae } & Ferraria crispa Burm. & Black flag, starfish iris & & Rubus fruticosus L. (species & European blackberry \\
\hline & \multirow{3}{*}{$\begin{array}{l}\text { Freesia leichtlinii F.W. Klatt [= F. alba } \\
\text { (G.L. Mey.) Gumbl. x F. Leichtlinii] } \\
\text { Moraea miniata Andrews }\end{array}$} & Freesia & & aggregate) & \\
\hline & & & \multirow{4}{*}{$\begin{array}{l}\text { Malaceae/ } \\
\text { Salicaceae }\end{array}$} & Populus x canescens (Aiton) Sm. & Gray poplar \\
\hline & & Two-leaf Cape tulip & & & \\
\hline Lamiaceae & Plectranthus comosus Sims. & Woolly coleus & & Populus deltoides Marshall & Common cottonwood \\
\hline \multirow[t]{5}{*}{ Liliaceae } & $\begin{array}{l}\text { Agapanthus praecox Willd. subsp. } \\
\text { orientalis (F.M. Leight.) F.M. Leight. }\end{array}$ & African lily, lily-of-the-nile & & Salix cinerea L. & $\begin{array}{l}\text { Large gray willow, pussy } \\
\text { willow }\end{array}$ \\
\hline & Alstroemeria aurea Graham & Peruvian-lily, alstroemeria & Scrophulariaceae & Scrophularia auriculata L. & Shoreline figwort \\
\hline & \multirow{2}{*}{$\begin{array}{l}\text { Asparagus densiflorus (Kunth) } \\
\text { Jessop }\end{array}$} & \multirow{2}{*}{$\begin{array}{l}\text { Sprenger's asparagus } \\
\text { fern }\end{array}$} & \multirow[t]{3}{*}{ Solanaceae } & Cestrum aurantiacum Lindl. & Orange jessamine \\
\hline & & & & Physalis peruviana L. & Peruvian groundcherry \\
\hline & Gloriosa superba L. & Glory lily, flame lily & & Solanum pseudocapsicum L. & Jerusalem-cherry \\
\hline Meliaceae & Azadirachta indica A. Juss. & Neem & Ulmaceae & Celtis sinensis Pers. & Chinese hackberry \\
\hline Myrsinaceae & Ardisia crenata Sims & Hen's eyes & \multirow[t]{4}{*}{ Verbenaceae } & \multirow{3}{*}{$\begin{array}{l}\text { Glandularia pulchella (Sweet) } \\
\text { Troncoso (= Verbena tenuisecta } \\
\text { Briq.) }\end{array}$} & \multirow{3}{*}{$\begin{array}{l}\text { South American mock } \\
\text { vervain }\end{array}$} \\
\hline \multirow[t]{5}{*}{ Myrtaceae } & Eucalyptus conferruminata & Bushy yate & & & \\
\hline & Eugenia uniflora $\mathrm{L}$. & Surinam cherry & & & \\
\hline & Psidium cattleianum Sabine & Strawberry guava & & Stachytarpheta spp. & Snakeweed \\
\hline & Psidium guajava $\mathrm{L}$. & Guava & Vitaceae & Vitis riparia Michx. & Riverbank grape \\
\hline & $\begin{array}{l}\text { Syzygium paniculatum Gaertn.(= } \\
\text { Eugenia myrtifolia Sims) }\end{array}$ & Brush cherry & \multirow[t]{3}{*}{ Zingiberaceae } & $\begin{array}{l}\text { Alpinia zerumbet (Pers.) B.L. Burtt. } \\
\text { \& R.M. Sm. }\end{array}$ & Shellplant \\
\hline \multirow[t]{2}{*}{ Oleaceae } & Ligustrum sinense Lour. & Chinese privet & & Hedychium coronarium J. Koenig & White ginger, garland-lily \\
\hline & Ligustrum vulgare $\mathrm{L}$. & European privet & & $\begin{array}{l}\text { Hedychium flavescens Carey ex } \\
\text { Roscoe }\end{array}$ & $\begin{array}{l}\text { Yellow ginger lily, cream } \\
\text { garland-lily }\end{array}$ \\
\hline Onagraceae & Oenothera drummondii Hook. & Beach eveningprimrose & \multicolumn{3}{|c|}{ Based on Brenzel (2007) or Hill and Narizny (2004). } \\
\hline Papaveraceae & Argemone ochroleuca Sweet & $\begin{array}{l}\text { Pale Mexican } \\
\text { pricklypoppy }\end{array}$ & \multicolumn{3}{|c|}{ * These should be reviewed by the horticulture industry and also watched for any spread into wildlands. } \\
\hline
\end{tabular}


TABLE 3. Plants already naturalized in California, but not sold as ornamentals in California

\begin{tabular}{lll}
\hline \hline Family & Species & Common name \\
\hline Asteraceae & Chrysanthemoides monilifera (L.) Norlindh & Boneseed, bitou bush \\
Boraginaceae & Heliotropium amplexicaule Vahl & Clasping or blue heliotrope \\
Fabaceae & Acacia paradoxa DC. & Kangaroothorn \\
\hline Acacia pycnantha Benth. & Golden wattle \\
Iridaceae & Cytisus multiflorus (L'Hér.) Sweet & White spanishbroom \\
Poaceae & Romulea rosea (L.) Eckl. & Rosy sandcrocus \\
Polygonaceae & Agrostis capillaris L. & Colonial bentgrass \\
Rosaceae & Polygonum aviculare L. & Prostrate knotweed \\
Solanaceae & Rubus ulmifolius Schott & Elmleaf blackberry \\
\hline
\end{tabular}

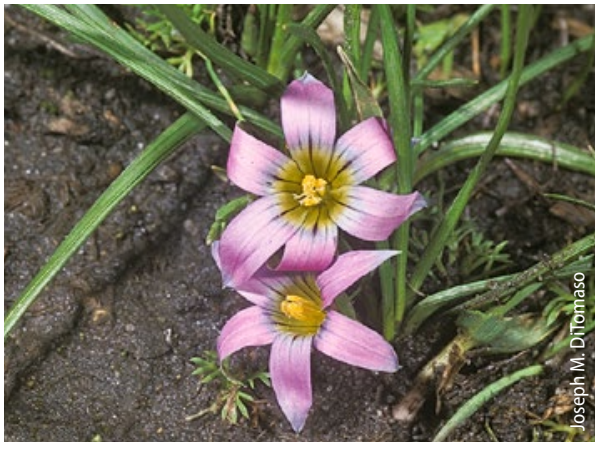

Rosy sandcrocus (Romulea rosea), a fairly new invasive species along the central coast of California, was introduced as a garden ornamental.

TABLE 4. Plants naturalized in California and also sold as ornamentals in the state*

\begin{tabular}{|c|c|c|c|c|c|}
\hline Family & Species & Common name & Family & Species & Common name \\
\hline Aizoaceae & Malephora crocea (Jacq.) Schwantes & $\begin{array}{l}\text { Coppery } \\
\text { mesembryanthemum }\end{array}$ & Iridaceae & $\begin{array}{l}\text { Chasmanthe floribunda (Salisb.) } \\
\text { N.E. Br. }\end{array}$ & African cornflag \\
\hline \multirow[t]{3}{*}{ Apocynaceae } & \multirow[t]{2}{*}{ Catharanthus roseus (L.) G. Don } & \multirow{2}{*}{$\begin{array}{l}\text { Pink periwinkle, } \\
\text { Madagascar periwinkle }\end{array}$} & \multirow[t]{2}{*}{ Lamiaceae } & Lavandula stoechas L. & French lavender \\
\hline & & & & Salvia verbenaca $\mathrm{L}$. & Wild clary \\
\hline & Nerium oleander L. & Oleander & Malvaceae & Hibiscus trionum $\mathrm{L}$. & Venice mallow \\
\hline Aponogetonaceae & Aponogeton distachyos L. f. & Cape pondweed & Myrtaceae & Eucalyptus cladocalyx F. Muell. & Sugargum \\
\hline Arecaceae & Phoenix dactylifera L. & Date palm & \multirow[t]{2}{*}{ Oleaceae } & Ligustrum japonicum Thunb. & Japanese privet \\
\hline $\begin{array}{l}\text { Asphodelaceae } \\
\text { (formerly Liliaceae) }\end{array}$ & Kniphofia uvaria (L.) Oken & Redhot poker & & Ligustrum lucidum Ait. & Glossy privet \\
\hline \multirow{8}{*}{ Asteraceae } & \multirow{3}{*}{$\begin{array}{l}\text { Coreopsis lanceolata L. } \\
\text { Erigeron karvinskianus DC. }\end{array}$} & \multirow{2}{*}{ Garden coreopsis } & \multirow{2}{*}{$\begin{array}{l}\text { Onagraceae } \\
\text { Oxalidaceae }\end{array}$} & Fuchsia magellanica Lam. & Hardy fuchsia \\
\hline & & & & Oxalis latifolia Kunth & Broadleaf woodsorrel \\
\hline & & $\begin{array}{l}\text { Mexican daisy, Latin } \\
\text { American fleabane }\end{array}$ & Papaveraceae & Papaver somniferum L. & Opium poppy \\
\hline & Gazania linearis (Thunb.) Druce & Treasureflower & \multirow[t]{4}{*}{ Passifloraceae } & Passiflora tarminiana Coppens \& & Banana passionfruit \\
\hline & Helianthus tuberosus L. & Jerusalem artichoke & & V.E. Barney & \\
\hline & Osteospermum ecklonis (DC.) Norl. & African daisy & & $\begin{array}{l}\text { Passiflora tripartita (Juss.) Poir. var. } \\
\text { mollissima (Kunth) Holm-Niesen \& }\end{array}$ & Banana passionfruit \\
\hline & \multirow[t]{2}{*}{ Osteospermum fruticosum (L.) Norl. } & \multirow{2}{*}{$\begin{array}{l}\text { Trailing African daisy, } \\
\text { shrubby daisybush }\end{array}$} & & P.M. Jerg. & \\
\hline & & & \multirow[t]{2}{*}{ Pinaceae } & Pinus halepensis Mill. & Aleppo pine \\
\hline Berberidaceae & Berberis darwinii Hook. & Darwin's berberis & & Pinus pinea $\mathrm{L}$. & Italian stone pine \\
\hline \multirow[t]{3}{*}{ Bignoniaceae } & Jacaranda mimosifolia D. Don & Jacaranda, black poui & \multirow[t]{4}{*}{ Pittosporaceae } & Pittosporum tobira (Thunb.) W.T. & Mock orange, Japanese \\
\hline & \multirow{2}{*}{$\begin{array}{l}\text { Macfadyena unguis-cati (L.) A. } \\
\text { Gentry }\end{array}$} & \multirow{2}{*}{$\begin{array}{l}\text { Cat's claw creeper, } \\
\text { catclaw-vine }\end{array}$} & & Aiton & cheesewood \\
\hline & & & & Pittosporum undulatum Vent. & Sweet pittosporum, \\
\hline Cabombaceae & Cabomba caroliniana Gray & Fanwort & & & Victorian box \\
\hline Caprifoliaceae & Lonicera japonica Thunb. & Japanese honeysuckle & Poaceae & Eragrostis curvula (Schrader) Nees & Weeping lovegrass \\
\hline Celastraceae & Maytenus boaria Molina & Mayten & & Pennisetum ciliare (L.) Link & Buffelgrass \\
\hline \multirow[t]{3}{*}{ Clusiaceae } & Hypericum androsaemum L. & Sweet-amber & Polygalaceae & Polygala myrtifolia L. & Myrtle-leaf milkwort \\
\hline & \multirow[t]{2}{*}{ Hypericum calycinum L. } & \multirow{2}{*}{$\begin{array}{l}\text { Aaron's beard, rose of } \\
\text { Sharon }\end{array}$} & Proteaceae & Grevillea robusta A. Cunn. ex R. Br. & Silkoak \\
\hline & & & Ranunculaceae & Clematis vitalba $\mathrm{L}$. & Old-man's-beard \\
\hline Convolvulaceae & Ipomoea indica (Burm. f.) Merr. & Blue morningglory & Rosaceae & Eriobotrya japonica (Thunb.) Lindl. & Loquat \\
\hline Ebenaceae & Diospyros lotus L. & Persimmon, date plum & & Rosa canina $\mathrm{L}$. & Dog rose \\
\hline \multirow[t]{8}{*}{ Fabaceae } & Acacia baileyana F. Muell. & $\begin{array}{l}\text { Bailey acacia, } \\
\text { cootamundra wattle }\end{array}$ & & $\begin{array}{l}\text { Rosa eglanteria L. (= Rosa rubiginosa } \\
\text { L.) }\end{array}$ & Sweetbriar rose \\
\hline & Acacia elata A. Cunn. ex Benth. & Cedar wattle & Salicaceae & Populus nigra L. var. italica DuRoi. & Black poplar, Lombardy \\
\hline & Dipogon lignosus (L.) Verdc. & Okie bean & & & popular \\
\hline & Gleditsia triacanthos L. & Honey locust & Solanaceae & Datura inoxia P. Mill. & Pricklyburr \\
\hline & Senna didymobotrya (Fresen.) Irwin & African senna & Tamaricaceae & Tamarix chinensis Lour. & Five-stamen tamarisk \\
\hline & \& Barneby & & Ulmaceae & Ulmus parvifolia Jacq. & Chinese elm \\
\hline & $\begin{array}{l}\text { Senna multiglandulosa (Jacq.) Irwin } \\
\text { \& Barneby }\end{array}$ & Glandular senna & & Ulmus pumila L. & Siberian elm \\
\hline & Geranium lucidum & Shining geranium & Verbenaceae & Lantana camara L. & Lantana \\
\hline Geramaceat & Geranium robertianum L. & Herb-robert & $\begin{array}{l}{ }^{*} \text { These may be con } \\
\text { and also watched }\end{array}$ & $\begin{array}{l}\text { d for removal from the trade through discussion } \\
\text { ither spread into wildlands. }\end{array}$ & Is with the horticulture industry \\
\hline
\end{tabular}



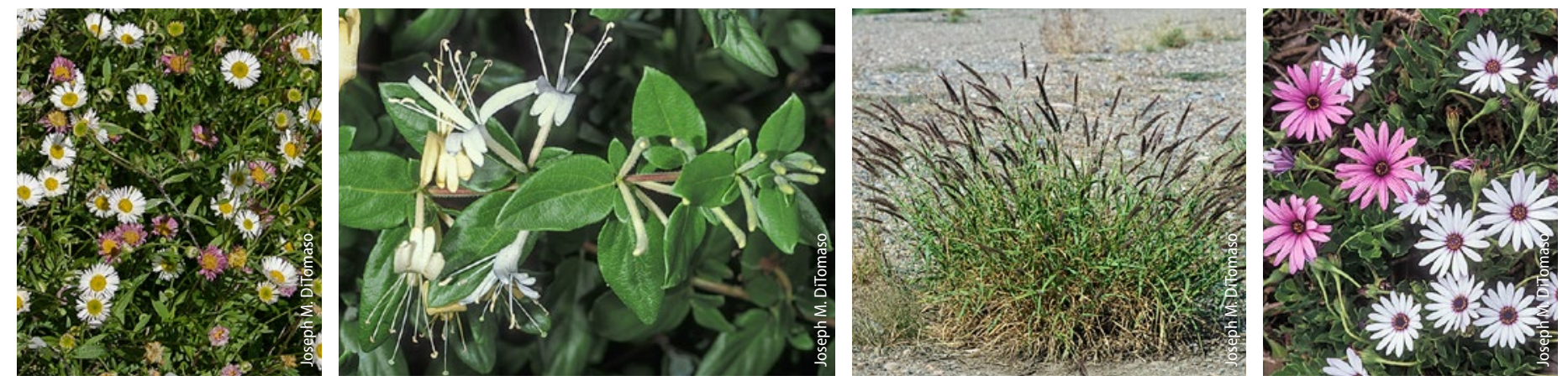

Species introduced as ornamentals or forage species that have escaped cultivation in California include, left, Mexican daisy (Erigeron karvinskianus), Japanese honeysuckle (Lonicera japonica), buffelgrass (Pennisetum ciliare) and African daisy (Osteospermum ecklonis). While these species are not yet major problems in the state, some have become more serious invasive plants in other regions of the country.

full prescreening risk assessment before introduction to the state. Plants that are not naturalized in California but that are sold here (table 2) should be reviewed by the nursery industry to reduce their sale and also watched for any spread into wildlands. In addition, noninvasive ornamentals that serve the same purpose in a landscape (same plant shape, same color flowers, etc.) should be promoted as alternative options. Species that are naturalized but not yet sold in California (table 3) should be restricted from sale, and land managers should watch for their further spread. Finally, species that are both naturalized and also sold in California (table 4) may be considered for removal from the trade and also watched by land managers for further spread into wildlands.

This list provides a good starting point for identifying plants, especially ornamental species, that are invasive in regions with similar climates to California and could become problematic here.

However, additional steps are required to further understand the potential risk of invasion. In particular, a more detailed risk assessment should be conducted for each of the species we identified as being at high risk for future invasion. Several risk assessment protocols (e.g., DiTomaso et al. 2012; Koop et al. 2012; Reichard and Hamilton 1997) are available to prioritize the greatest potential threats to wildland systems. Implementing these preventative approaches and establishing an early detection program to eradicate incipient populations of these targeted species are far less costly than attempting to manage or contain large well-established populations of invasive plants.

E.D. Brusati is Senior Scientist, California Invasive Plant Council (Cal-IPC), Berkeley; D.W. Johnson is Executive Director, Cal-IPC, Berkeley; and J.M. DiTomaso is UC Cooperative Extension Specialist, UC Davis.

This work was supported by a grant from the UC Integrated Pest Management Program. We thank B. McKinley for her help with this project and two anonymous reviewers for their comments.

\section{References}

Baldwin BG, Goldman DH, Keil DJ, et al. (eds.). 2012. The Jepson Manual: Vascular Plants of California (2nd ed.). Berkeley, CA: UC Press.

Bell CE, DiTomaso JM, Wilen CA. 2007. Invasive Plants: Integrated Pest Management for Home Gardeners and Landscape Professionals. UC ANR Pub 74139. Oakland, CA. 7 p.

Bradley BA, Oppenheimer M, Wilcove DS. 2009. Climate change and plant invasions: Restoration opportunities ahead? Global Change Biol 15:1511-21. doi:10.1111/ j.1365-2486.2008.01824.x

Brenzel KN. 2007. Sunset Western Garden Book. Menlo Park, CA: Sunset Publishing.

Caley P, Kuhnert DS. 2006. Application and evaluation of classification trees for screening unwanted plants. Austral Ecol 31:647-55. doi:10.1111/j.1442-9993.2006.01617.x.

[Cal-IPC] California Invasive Plant Council. 2013. California Invasive Plant Inventory. Cal-IPC Publication 2006-02 (and subsequent updates). Berkeley, CA. www.cal-ipc.org.

[CDFA] California Department of Food and Agriculture. 2007. Pest Ratings of Noxious Weed Species and Noxious Weed Seed. State of California, Department of Food and Agriculture, Division of Plant Health and Pest Prevention Services. Sacramento, CA. January 2007.

CDFA. 2012. California County Agricultural Commissioner's Reports, 2011. California Department of Food and Agriculture. Sacramento, CA. www.nass.usda.gov/ Statistics_by_State/California/Publications/AgComm/ Summary/index.asp.
CDFA. 2013. Weed Eradication: Program Details. State of California, Department of Food and Agriculture, Division of Plant Health and Pest Prevention Services. www.cdfa. ca.gov/plant/ipc/weeds/weeds_hp.htm.

Dehren-Schmutz K, Touza J, Perrings C, Williamson M. 2007. A century of the ornamental plant trade and its impact on invasion success. Divers Distrib 13:527-34. doi:10.1111/j.1365-2664.2011.02061.x

DiTomaso JM, Conser C, Seebacher L, Brush R. 2012. The development and validation of a more accurate weed risk assessment tool for evaluating the invasive potential of ornamental plants. In: Proc California Invasive Plant Council Symposium, Oct. 11-12, 2012. Rohnert Park, CA. www.cal-ipc.org/symposia/archive/2012_presentations. php.

DiTomaso JM, Healy EA. 2007. Weeds of California and Other Western States. UC ANR Pub 3488, 2 Vols. Oakland, CA. $1808 \mathrm{p}$.

Drew J, Anderson N, Andow D. 2010. Conundrums of a complex vector for invasive species control: A detailed examination of the horticultural industry. Biol Invasions 12:2837-51. doi:10.1007/s10530-010-9689-8.

Hill S, Narizny S. 2004. The Plant Locator: Western Region. Portland, OR: Black Susan Press.

Koop AL, Fowler L, Newton LP, Caton BP. 2012. Development and validation of a weed screening tool for the United States. Biol Invasions 14:273-94. doi:10.1007/ s10530-011-0061-4.
Nevada Department of Agriculture. 2005. Noxious Weed List. Nevada Department of Agriculture, Plant Industry Division. Reno, NV. http://agri.nv.gov/Plant/Noxious_ Weeds/Noxious_Weed_List/.

Northam FE, Backer DM, Hall JA. 2005. Development of a Categorized List of Invasive Non-Native Plants That Threaten Wildlands in Arizona. Southwestern Vegetation Management Association. www.swvma.org/DevelopmentOfACategorizedList.pdf.

Okada M, Ahmad R, Jasieniuk MA. 2007. Microsatellite variation point to local landscape plantings as sources of invasive pampas grass (Cortaderia selloana) in California. Mol Ecol 16:4956-71. doi:10.1111/j.1365294X.2007.03568.x

Oregon Department of Agriculture. 2006. State Noxious Weed List. Salem, OR. www.oregon.gov/ODA/PLANT/ WEEDS/pages/lists.aspx.

Reichard SH, Hamilton CW. 1997. Predicting invasions of woody plants introduced into North America. Conserv Biol 11:193-203.

Reichard SH, White P. 2001. Horticulture as a pathway of invasive plant introductions in the United States. Bioscience 51:103-13. 